\title{
Severe Ulnar Nerve Injury After Bee Venom Acupuncture at a Traditional Korean Medicine Clinic: A Case Report
}

\author{
Joon Sang Park, MD ${ }^{1,2}$, Yoon Ghil Park, MD, PhD ${ }^{1,2}$, Chul Hoon Jang, MD ${ }^{1,2}$, \\ Yoo $\mathrm{Na}$ Cho, $\mathrm{MD}^{1,2}$, Jung Hyun Park, $\mathrm{MD}, \mathrm{PhD}^{1,2}$ \\ ${ }^{1}$ Department of Rehabilitation Medicine, Gangnam Severance Hospital, Seoul; \\ ${ }^{2}$ Rehabilitation Institute of Neuromuscular Disease, Yonsei University College of Medicine, Seoul, Korea
}

\begin{abstract}
This case report describes a severe nerve injury to the right ulnar nerve, caused by bee venom acupuncture. A 52-year-old right-handed man received bee venom acupuncture on the medial side of his right elbow and forearm, at a Traditional Korean Medicine (TKM) clinic. Immediately after acupuncture, the patient experienced pain and swelling on the right elbow. There was further development of weakness of the right little finger, and sensory changes on the ulnar dermatome of the right hand. The patient visited our clinic 7 days after acupuncture. Electrodiagnostic studies 2 weeks after the acupuncture showed ulnar nerve damage. The patient underwent steroid pulse and rehabilitation treatments. However, his condition did not improve completely, even 4 months after acupuncture.
\end{abstract}

Keywords Bee venoms, Ulnar neuropathy, Korean traditional medicine

\section{INTRODUCTION}

An estimated $86 \%$ Korean residents have availed some form of Traditional Korean Medicine (TKM) at least once in their lifetime, with maximum application of acupuncture [1]. In particular, bee venom acupuncture is a commonly practiced treatment for musculoskeletal and joint pain in TKM [2]. Although focal inflammation is a minor and temporary side effect of bee venom acupuncture [3], severe complications can arise when applied around or directly to a nerve. This case report is the first to describe severe ulnar nerve injury caused by bee venom acupuncture, performed at a TKM clinic.

\section{CASE REPORT}

In April 2015, a 52-year-old right-handed man visited our clinic presenting with a right claw hand, decreased

Received May 19, 2016; Accepted August 10, 2016

Corresponding author: Jung Hyun Park

Department of Rehabilitation Medicine, Gangnam Severance Hospital, 211 Eonju-ro, Gangnam-gu, Seoul 06273, Korea. Tel: +82-2-2019-3491, Fax: +82-2-3463-7585, E-mail: RMPJH@yuhs.ac

ORCID: Joon Sang Park (http://orcid.org/0000-0002-0025-9704); Yoon Ghil Park (http://orcid.org/0000-0001-9054-5300); Chul Hoon Jang (http:// orcid.org/0000-0001-9432-3473); Yoo Na Cho (http://orcid.org/0000-0002-8792-5828); Jung Hyun Park (http://orcid.org/0000-0003-3262-7476).

(c) This is an open-access article distributed under the terms of the Creative Commons Attribution Non-Commercial License (http://creativecommons.org/ licenses/by-nc/4.0) which permits unrestricted noncommercial use, distribution, and reproduction in any medium, provided the original work is properly cited. Copyright (c) 2017 by Korean Academy of Rehabilitation Medicine 
sensation below the right elbow, and decreased motor strength in the 4th and 5th digits of the hand. Previously, the patient had pain in the right elbow, and had received more than 10 bee venom acupunctures on the medial side of the affected elbow and forearm, at a TKM clinic. Immediately after the procedure, he experienced severe pain and swelling of the right elbow. Although the pain subsided several days after the acupuncture, he had developed weakness of the right 4th and 5th finger flexors, and a sensory change on the ulnar dermatome of the right hand. When the patient was referred to our clinic 7 days after acupuncture, the strength of the 3rd and 4th lumbricals and interosseous muscles of the right hand were graded 1 out of 5 by a numeric rating scale.

A right ulnar motor nerve conduction study 2 weeks after the injury, showed low amplitude of compound muscle action potentials and slow nerve conduction velocity at the elbow segments, indicating potential focal segmental demyelination, axonal damage, and Wallerian degeneration.

A right ulnar sensory nerve conduction study showed low amplitude of sensory nerve action potentials (SNAPs) (Table 1). Electromyography revealed abnormal spontaneous activity and a decreased interference pattern at the right abductor digiti minimi muscle and the first dorsal interosseous muscles, and abnormal spontaneous activity at the right flexor carpi ulnaris (Table 2). The patient underwent steroid pulse therapy for 4 weeks since his first visit, and also received electrical stimulation therapy at an outpatient clinic.

Table 1. Results of the nerve conduction study

\begin{tabular}{|c|c|c|c|c|c|c|}
\hline & Nerve & DSL (m/s) & S Amp $(\mu V)$ & DML (ms) & M Amp (mV) & $\mathrm{NCV}(\mathrm{m} / \mathrm{s})$ \\
\hline \multirow[t]{8}{*}{14 days from onset } & Rt. median & 2.7 & 38.2 & 2.5 & 12.5 & 63.5 \\
\hline & Rt. ulnar & 2.3 & 7.7 & 2.6 & 0.4 (wrist) & 50.6 (BE-wrist) \\
\hline & & & & & $0.3(\mathrm{BE})$ & $41.0(\mathrm{AE}-\mathrm{BE})$ \\
\hline & & & & & $0.2(\mathrm{AE})$ & \\
\hline & Rt. dorsal ulnar & 1.3 & 1.6 & & & \\
\hline & Lt. median & 2.4 & 49.4 & 2.7 & 15.6 & 65.1 \\
\hline & Lt. ulnar & 2.0 & 21.5 & 2.3 & 15.0 & 72.5 \\
\hline & Lt. dorsal ulnar & 1.3 & 19.0 & & & \\
\hline \multirow[t]{5}{*}{135 days from onset } & Rt. ulnar & 2.4 & 8.7 & 2.9 & 1.3 (wrist) & 51.9 (BE-wrist) \\
\hline & & & & & $1.2(\mathrm{BE})$ & 35.7 (AE-BE) \\
\hline & & & & & $1.1(\mathrm{AE})$ & \\
\hline & Rt. dorsal ulnar & No response & No response & & & \\
\hline & Lt. dorsal ulnar & 1.4 & 20.0 & & & \\
\hline
\end{tabular}

DSL, distal sensory latency; S Amp, sensory amplitude; DML, distal motor latency; M Amp, motor amplitude; NCV, nerve conduction velocity; $\mathrm{BE}$, below elbow; $\mathrm{AE}$, above elbow.

Table 2. Results of needle electromyography

\begin{tabular}{llccc}
\hline & Muscle & $\begin{array}{c}\text { Rest activity } \\
\text { PSW/fibrillation }\end{array}$ & Recruitment & Volitional activity \\
\hline 14 days from onset & Rt. ADM & $2+$ & Reduced & NMU \\
& Rt. FCU & $2+$ & Normal & NMU \\
135 days from onset & Rt. 1st DI & $2+$ & Reduced & NMU \\
& Rt. ADM & $2+$ & Reduced & Giant, LPW \\
& Rt. FCU & 0 & Normal & Giant, LPW \\
\hline
\end{tabular}

PSW, positive sharp wave; ADM, abductor digiti minimi; NMU, normal motor unit; FCU, flexor carpi ulnaris; 1st DI, first dorsal interosseous; LPW, long duration polyphasic wave. 
Magnetic resonance images of the right forearm, hand, and wrist were obtained 1 month after the injury. Swelling of the right ulnar nerve at the cubital tunnel was observed (Fig. 1). Ultrasonography showed diffuse right ulnar nerve swelling, and the cross-sectional area in transverse view at elbow level was $13 \mathrm{~mm}^{2}$. On the other hand, left side cross-sectional area was $8 \mathrm{~mm}^{2}$. There was subluxation of the right ulnar nerve escaping the cubital tunnel to the anterolateral area of the forearm during elbow flexion (Fig. 2). An electrodiagnostic study was con-
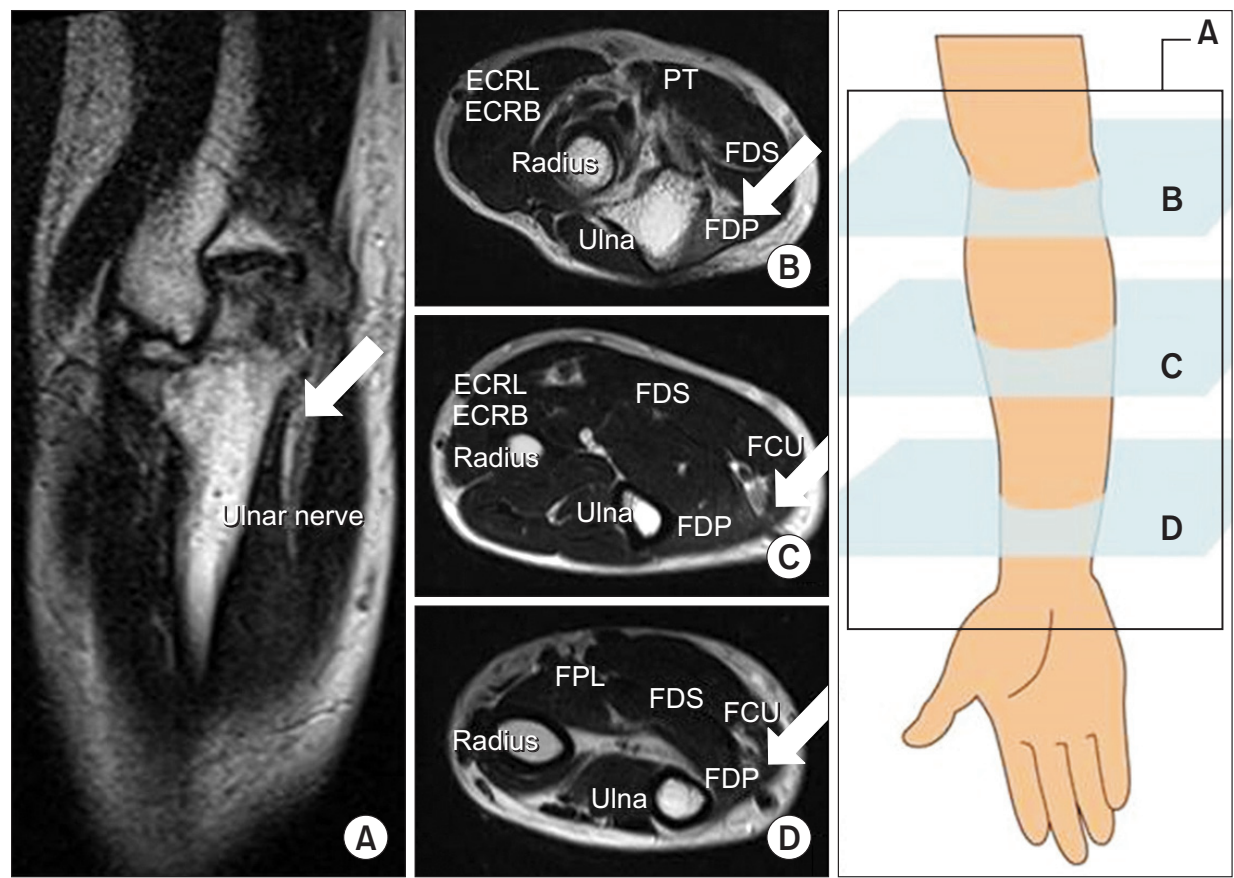

Fig. 1. Magnetic resonance images of the right elbow and forearm. Diffuse swelling and inflammation of muscles innervated by the ulnar nerve are apparent. Arrows show edema and inflammatory changes in the right elbow and forearm. (A) T2 coronal view: at the elbow level, arrow indicates ulnar nerve. (B-D) T2 transverse views: at the level of the proximal forearm, arrow indicates FDP (B); distal forearm, arrow indicates FCU (C); and wrist, arrow indicates FCU (D). ECRB, extensor carpi radialis brevis; ECRL, extensor carpi radialis longus; FCU, flexor carpi ulnaris; FDP, flexor digitorum profundus; FDS, flexor digitorum superficialis; FPL, flexor pollicis longus; PT, pronator teres.
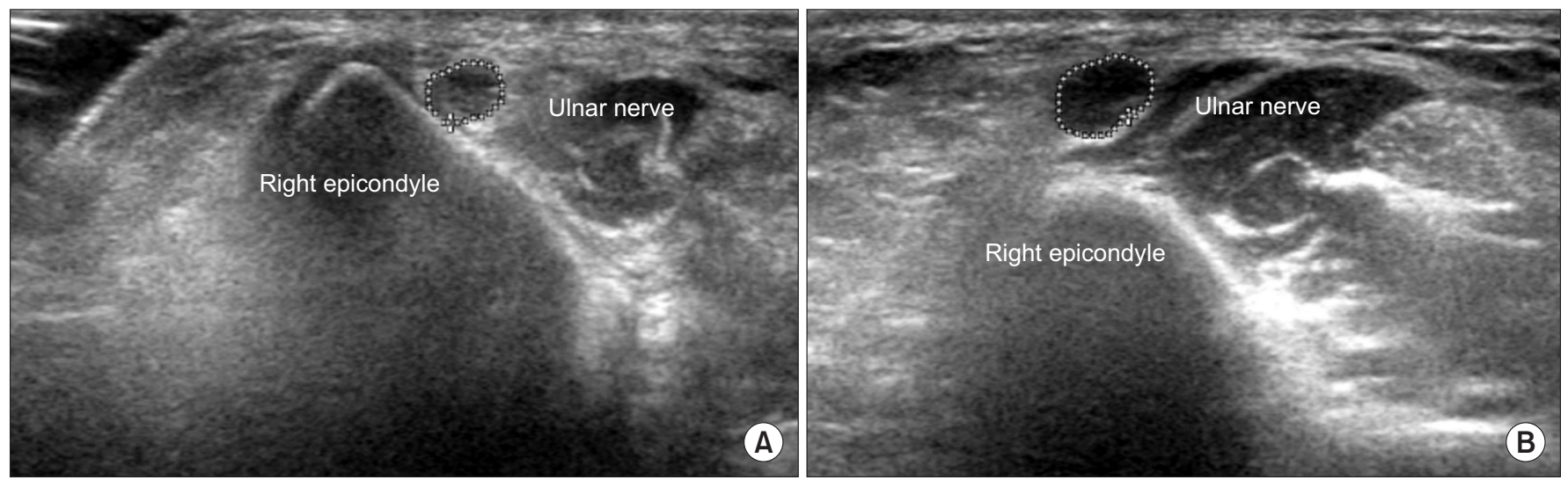

Fig. 2. Subluxation of the ulnar nerve. Ultrasonographic images of the ulnar nerve showing subluxation at the elbow level during elbow flexion. (A) The dotted area indicates the ulnar nerve, which is in a neutral position during elbow extension. (B) Nerve subluxation during elbow flexion. 
ducted 4 months after the injury. There was decreased right ulnar motor conduction velocity at the elbow segment and no response of SNAP at the right dorsal ulnar nerve (Table 1). A giant and long duration polyphasic motor unit was found in the right forearm and hand muscles innervated by the ulnar nerve (Table 2). Thus, although there was evidence of regeneration of the denervated muscles, complete recovery was not indicated by the electrodiagnostic study (Table 1).

\section{DISCUSSION}

This case report highlights the concern regarding medical complications arising from TKM treatment. Acupuncture points most commonly used for elbow pain are located on the medial side of right elbow near the ulnar nerve course (arrow in Fig. 3) [4]. However, the patient showed subluxation of the ulnar nerve escaping the cubital tunnel to the anterolateral area of the forearm during elbow flexion (Fig. 2), which overlapped the course of the acupuncture points. This implies that the ulnar nerve injury was likely caused by focal inflammation or by direct injury of bee venom acupuncture around or direct to the ulnar nerve. Bee venom acupuncture is more dangerous than needle acupuncture if done around or directly to the nerve [5]; this observation is supported by the sustained

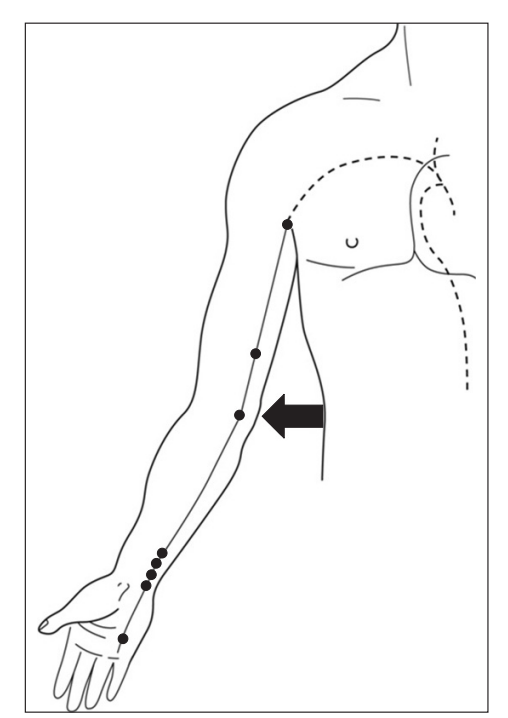

Fig. 3. Acupuncture diagram. The dots indicate the course of the acupoints, known as the heart meridian of the hand-shaoyin, and are near, but not the same as, the ulnar nerve course. nerve swelling in magnetic resonance imaging and ultrasonography 1 month after acupuncture in this case. Further studies are required to understand the neurotoxic effects of bee venom more exhaustively.

To avoid nerve damage, TKM practitioners should know the exact anatomical positions of the nerves. The physical examination and an electrodiagnostic study revealed the lack of a complete recovery from the ulnar nerve injury. Thus, the patient sustained poor performance of daily activities by severe dysfunction of his right hand.

Traditional medical practices are common. For example, the percentage of people who have used some form of traditional medicine is $68.9 \%$ in Australia, $90 \%$ in China, $86 \%$ in Korea, and 53\% in Singapore [6,7]. However, there are few published reports relating to the adverse effects of TKM treatments [8,9]. This is the first report of ulnar nerve injury as a complication of bee venom acupuncture, and indicates the possibility of sustained complications from TKM procedures, which may be avoidable by the increased anatomical training of practitioners.

\section{CONFLICT OF INTEREST}

No potential conflict of interest relevant to this article was reported.

\section{REFERENCES}

1. Korea Institute of Oriental Medicine. A survey on the use of traditional Korean medicine, 2008. Seoul: Ministry of Health and Welfare; 2009.

2. Lee JD, Park HJ, Chae Y, Lim S. An overview of bee venom acupuncture in the treatment of arthritis. Evid Based Complement Alternat Med 2005;2:79-84.

3. Chen J, Lariviere WR. The nociceptive and anti-nociceptive effects of bee venom injection and therapy: a double-edged sword. Prog Neurobiol 2010;92:151-83.

4. Schroeder S, Meyer-Hamme G, Zhang J, Epplee S, Friedemann $\mathrm{T}, \mathrm{Hu} \mathrm{W}$. An acupuncture research protocol developed from historical writings by mathematical reflections: a rational individualized acupoint selection method for immediate pain relief. Evid Based Complement Alternat Med 2013;2013:256754.

5. White A. A cumulative review of the range and incidence of significant adverse events associated with acupuncture. Acupunct Med 2004;22:122-33. 
6. World Health Organization. The regional strategy for traditional medicine in the Western Pacific (20112020). Manila: World Health Organization, Regional Office for the Western Pacific; 2002.

7. Korea Ministry of Health and Welfare. A survey on the use of traditional Korean medicine and herbal medicine. Seoul: Ministry of Health and Welfare; 2011.
8. Ha SY, Kang KW. Two cases of lead poisoning after taking herb pills, Hwangdan (Red Lead). J Korean Pediatr Soc 1979;22:64-70.

9. Kim Y, Kim Y, Lee H. The clinical study on 6 cases of patients with side effect caused by acupuncture therapy. J Daejeon Univ Tradit Korean Med Inst 2006;15:4752. 